\title{
On the use of Frequency-Domain Subspace-based System Identification for Estimating Resonant Systems
}

\author{
Lucas F. M. Rodrigues* Gustavo H. C. Oliveira* \\ Diogo J. D. E. Santo** \\ * Electrical Engineering Department, Federal University of Parana, \\ Curitiba-PR, (e-mail: fariaslucasrodrigues@ufpr.br and \\ gustavo@eletrica.ufpr.br). \\ ** ESBR-Energia Sustentável do Brasil, Porto Velho- RO, (e-mail: \\ diogo.santo@esbr.com.br)
}

\begin{abstract}
In this paper, a parametric identification of high order resonant systems from frequency response data is analyzed. Among many techniques for obtaining black-box models for such systems, subspace-based algorithms stand out. Subspace methods uses geometric projections to obtain specific information necessary to compute the system extended observability matrix and, eventually, to obtain a state-space model representation. Throughout the paper, the method is also shown to use only well conditioned matrices to avoid numerical problems with high order models. The validity of the algorithm is firstly demonstrated on a simulated data of a synthetic 15th order system. Then, in order to state the systems effectiveness, the method is applied to actual data extracted from a resonant beam.
\end{abstract}

Keywords: Black-Box Modeling, System Identification, Subspace-Based Algorithms, Resonant System, High voltage equipment

\section{INTRODUCTION}

Resonant systems and vibration analysis are object of many study areas in engineering and widely applied in structural analysis, civil construction, aeronautics, electrical engineering and others. Several recent scientific research about resonant systems are addressed willing to obtain detailed description of its models, to pattern its resonant poles and behavior (Jeong et al., 2013; Noshadi et al., 2016; Kumon et al., 2000). Indeed, due to the oscillatory nature of resonant systems, it is highly recommended to investigate their operational limits via reliable simulations in order to avoid disruptions and injuries to equipment. Therefore, procedures to obtain an accurate description of the system behavior is of great interest.

A considerable number of studies has focused in computing resonant systems models in the field of structural analysis, such as in El-Gazzar (2017); Dou and Jensen (2015); Ruzziconi et al. (2013). An important challenge facing researchers is that to investigate and monitor the effect of oscillations in the system's stability (Zhao et al., 2018; Yao and Li, 2015). Likewise, in the area of energy supply, given the growing complexity in operating power grids, to supervise the stability of the system became increasingly important. In a electrical power system, resonant equipment are interconnected in the network, and there is an ongoing power exchange among the components. In such

\footnotetext{
1 The authors would like to acknowledge grant from Energia Sustentável do Brasil - ESBR research and technological development program, through the $\mathrm{P} \& \mathrm{D}$ 06631.0006/2017 project, regulated by ANEEL.
}

cases, even small perturbations added to contingencies, can cause large power variations and lead to instability.

An equipment with resonant behavior that requires special attention are high voltage power transformers, due to its high cost equipment and importance to connect different parts of the grid (Cigrè-JWG-A2/C4.52, 2020; CigrèJWG-A2/C4.39, 2014). Brazilian study shows that for the 20 step-up transformers inspected due to failure, 6 of the failures were associated with resonant transients generated on the system (Bechara, 2010; Shipp et al., 2011). Moreover, literature shows that the effect of switching in Gas Insulated Substations (GIS) can damage connected power and instrument transformers due to the occurrence of Very Fast Transient Over-voltages (VFTO) (Gorayan and Chandrakar, 2013; Shu et al., 2013).

In this context, black-box models based on exogenous experimental data is an efficient procedure for describing dynamic behavior resonant system and requires little or no prior knowledge of the system to be modeled (Deschrijver et al., 2008; Grivet-Talocia and Gustavsen, 2016). However, obtaining a black-box model can be a complex and error-prone task at various stages of the process. Initially, a set of measurement data should be collected. Due to the properties of resonant systems, it is common in the electrical engineering to perform the data acquisition and system identification from frequency-domain data samples. Moreover, literature shows a frequent use of data analyzers to obtain information in frequency domain by collecting samples non-uniformly spaced (McKelvey, 1995; Ljung, 2007). Indeed, due to wide band nature of the measure- 
ment spam, the number of samples would be huge if the data was linearly spaced. Considering that, this paper is interested in system identification techniques based on non-uniformly spaced frequency-domain wide-band data.

Among noniterative methods to system identification, subspace-based algorithms have been the object of many research. It is a widely explored group whose applications are found in different fields as in Rongshan et al. (2014); Bakir (2011); Park and Noh (2011). This approach does not involve parametric optimization and is based on concepts from system theory, algebra and statistics (McKelvey et al., 1996).

When compared to traditional identification methodologies, this approach is advantageous because it requires no initial parametrization, but also due to its effortlessness on computing the state sequence directly from input-output data. Another point to highlight is the fact that there is no difference between SISO and MIMO identification for subspace-based algorithms. Furthermore, the idea of estimating one single state-space model in a minimal realization makes the technique attractive.

Although subspace system identification methods based on time-domain data are widely used in the literature, much less works addressing subspace methods for nonuniformly spaced frequency domain wide-band data are found, specially for resonant system study. Here, it should be mentioned the works of McKelvey et al. (1996) and Overschee and Moor (1996a). In light of that, this paper discusses the differences between the algorithms and their performance for resonant system applications. Therefore, this paper presents a comparison between both algorithms and its performance for resonant system applications.

This paper is organized as follows: in Section 2 the problem of estimating models from frequency domain data is formulated. Section 3 discusses two subspace system identification algorithms based on frequency domain non uniformly spaced data. In Section 4 , it is presented the application of the method in two case studies, the first one using data from a synthetic process and a second one considering an actual flexible beam.

\section{PROBLEM STATEMENT}

Consider a linear time-invariant, continuous-time system with $\mathrm{p}$ outputs and $\mathrm{m}$ inputs described by the following state-space equations:

$$
\begin{aligned}
& \dot{x}(t)=A x(t)+B u(t), \\
& y(t)=C x(t)+D u(t),
\end{aligned}
$$

with $u(t) \in R^{\mathrm{m}}$ and $y(t) \in R^{\mathrm{p}}$ input and output signals. Also assume that the system is of finite order $n$ and consider it initially relaxed.

Using Laplace transform, the time-domain description in (1) turns to a frequency-domain representation as in (2).

$$
\begin{aligned}
s X(s) & =A X(s)+B U(s), \\
Y(s) & =C X(s)+D U(s),
\end{aligned}
$$

where $s=j w$ is the Laplace complex variable. Eventually, the frequency response is given by

$$
Y(s)=D+C(s I-A)^{-1} B .
$$

When $U=\left(\begin{array}{llll}\mathbf{1} & \mathbf{1} \ldots \mathbf{1}\end{array}\right)$, a vector with ones, (2) is rewritten as (4). By making the input as mentioned we consider, at once, the information obtained from $m$ different input vectors. Note that $\bar{X}(s) \in R^{\mathrm{n} \times \mathrm{m}}$ is the transformed state vector caused by an impulse applied to the input.

$$
\begin{aligned}
s \bar{X}(s) & =A \bar{X}(s)+B I_{\mathrm{i}}, \\
Y(s) & =C \bar{X}(s)+D I_{\mathrm{i}} .
\end{aligned}
$$

Let $Y\left(j \omega_{k}\right)$ be the measured frequency response of an unknown device under test or equipment, where $\omega_{\mathrm{k}}$, $\mathrm{k}=1, \ldots, \mathrm{N}$, are the measured frequencies, which may not necessarily be equidistantly spaced and $\mathrm{N}$ is the number of samples. Then $\left\{Y\left(j \omega_{1}\right), \ldots, Y\left(j \omega_{\mathrm{N}}\right)\right\}$ is the set of samples.

Although equidistantly distributed data is widely applied in time-domain system identification, it is less common in practical applications for wide-band frequency-domain data. The use of equidistantly distributed data in logarithmic scale is a common tool to overcome the issues concerned to working with big sets of samples.

The problem addressed in this paper is the one to use subspace techniques to fit a state-space model in terms of the quadruple $(A, B, C, D)$, given a set of nonequidistantly spaced, wide-band, frequency domain samples $\left\{Y\left(j \omega_{1}\right), \ldots, Y\left(j \omega_{\mathrm{N}}\right)\right\}$.

\section{SUBSPACE-BASED IDENTIFICATION}

Subspace algorithms have been firstly formalized by Overschee and De Moor (1991) in which a novel algorithm to system identification by using stochastic models was proposed. Matter fact, this algorithm works well for systems of small order. Arguably, one may say that whereas the estimate order increases, the user faces some problems related to the conditioning number of some matrices, what may lead the procedure to undesired results. Latter, Overschee and Moor (1996b) have described more robust algorithms in their book, eliciting different approaches for time-domain system identification. After that, one can find many approaches of subspace methods in the literature. However, just a few authors have addressed their research to frequency-domain subspace-based identification using not equidistantly spaced data, as described in the previous section.

In this context, two works stand out: one, described in Overschee and Moor (1996a) whose objective is twofold: i) to present a simple frequency-domain system identification algorithm; $i i)$ to overcome the problem of estimating models of high order systems with better conditioned matrices. The second work was latter described in McKelvey et al. (1996) where the authors present two subspace based algorithms, a first procedure based on equidistantly, in frequency, spaced data, and a second one on non equidistantly spaced data.

The differences between the methods are to be highlighted in this paper. Lets begin stating that in McKelvey et al. (1996), the system identification methodology is designed for discrete-time systems, whereas in Overschee and Moor (1996a), a continuous-time frequency domain subspace system identification is developed. Because we are interested in continuous-time model estimation, this author 
transformed McKelvey et al. (1996) to a continuous-time approach.

\subsection{The Algorithm}

In general, subspace-based algorithms follow a few steps to obtain the system matrices $A, B, C$ and $D$. Initially, an output basis matrix is determined using the system response samples. Also, an input basis matrix is created usually from unit vectors somehow concatenated, depending on the approach. Then, an orthogonal projection is applied to the data matrices, which is latter decomposed via SVD. The SVD generates matrices $\left(U, S, V^{T}\right)$, where $(\cdot)^{T}$ denotes a transpose matrix, from which important information is obtained. At this point, it is either possible to estimate the order of the model, using the singular values information, and the extended observability matrix, what leads to compute the matrices $A$ and $C$. Lastly, $B$ and $D$ is determined through a least squares solution of a linear set of equations.

Step 1- Computing the data matrices Because we want to estimate high order models, we need to compute well conditioned input/output matrices. An efficient tool to overcome problems related to ill conditioned matrices are the Forsythe recursions, applied to determined the input basis $I_{F}$ and the output basis $Y_{F}$.

Let $D_{w}$ be a complex matrix with the measured frequencies, as follows

$$
D_{w}=\operatorname{diag}\left[\left(\left(j w_{1}\right)\left(j w_{2}\right) \ldots\left(j w_{N}\right) \otimes\left(\begin{array}{llll}
\mathbf{1} & \mathbf{1} & \ldots & \mathbf{1}
\end{array}\right)\right)\right]
$$

where $\otimes$ denotes a Kronecker product. Also assume the size of $\left(\begin{array}{llll}\mathbf{1} & \mathbf{1} & \ldots & \mathbf{1}\end{array}\right)$ as $p \times m$.

To find $Y_{F}$ and $I_{F}$, first initialize defining $R_{0}, R_{1}$ and $Z_{0}$, $Z_{1}$ as in (6) to (9). Lets denote the complex conjugated transpose as $(\cdot)^{*}$, and $\operatorname{diag}(\cdot)$ returns a square diagonal matrix with the elements on the main diagonal.

$$
\begin{gathered}
R_{0}=\left(Y\left(j w_{1}\right), Y\left(j w_{2}\right), \ldots, Y\left(j w_{\mathrm{N}}\right)\right), \\
Z_{0}:=\operatorname{diag}\left(\operatorname{diag}\left(R_{0} R_{0}^{*}\right)\right), \\
R_{1}:=R_{0} D_{\mathrm{w}}, \\
Z_{1}:=\operatorname{diag}\left(\operatorname{diag}\left(R_{1} R_{1}^{*}\right)\right) .
\end{gathered}
$$

Then, compute the recursion for $k=2$ to $q-1$, where $q$ is a user defined parameter corresponding to an initial estimate order.

$$
\begin{aligned}
R_{\mathrm{k}} & :=R_{\mathrm{k}-1} D_{\mathrm{w}}+\frac{Z_{\mathrm{k}-1}}{Z_{\mathrm{k}-2}} R_{\mathrm{k}-2} . \\
Z_{\mathrm{k}} & :=\operatorname{diag}\left(\operatorname{diag}\left(R_{\mathrm{k}} R_{\mathrm{k}}^{*}\right)\right) .
\end{aligned}
$$

Note that $R_{\mathrm{k}} \in \mathbf{C}^{q \times N}$ and $Z_{\mathrm{k}} \in \mathbf{C}^{q \times p}$. Now, defining $D_{1}$ and $D_{2}$, diagonal matrices of order $q-2$ as in (12) and (13), respectively.

$$
D_{1}=\left[\begin{array}{cccc}
\frac{Z_{1}^{1 / 2}}{Z_{2}^{1 / 2}} & 0 & \cdots & 0 \\
0 & \frac{Z_{2}^{1 / 2}}{Z_{3}^{1 / 2}} & \cdots & 0 \\
\cdots & \cdots & \cdots & \cdots \\
0 & 0 & \cdots & \frac{Z_{\mathrm{q}-2}^{1 / 2}}{Z_{\mathrm{q}-1}^{1 / 2}}
\end{array}\right],
$$

$$
D_{2}=\left[\begin{array}{cccc}
\frac{Z_{1}}{\left(Z_{0} Z_{2}\right)^{1 / 2}} & 0 & \cdots & 0 \\
0 & \frac{Z_{2}}{\left(Z_{1} Z_{3}\right)^{1 / 2}} & \cdots & 0 \\
\cdots & \cdots & \cdots & \cdots \\
0 & 0 & \cdots & \frac{Z_{q-2}}{\left(Z_{q-3} Z_{q-1}\right)^{1 / 2}}
\end{array}\right]
$$

Finally, the output basis, with the superscript $c$ standing for complex.

$$
Y_{\mathrm{F}}^{\mathrm{c}}=\left[\begin{array}{c}
\frac{R_{0}}{Z_{0}^{1 / 2}} \\
\frac{R_{1}}{Z_{1}^{1 / 2}} \\
\cdots \\
\frac{R_{\mathrm{q}-1}}{Z_{\mathrm{q}-1}^{1 / 2}}
\end{array}\right]
$$

Hence, $Y_{\mathrm{F}}=\left(\Re\left[Y_{\mathrm{F}}^{\mathrm{c}}\right] \Im\left[Y_{\mathrm{F}}^{\mathrm{c}}\right]\right)$, with $\Re(\cdot)$ and $\Im(\cdot)$ meaning the real and imaginary part of $(\cdot)$. Then, we have

$$
\begin{aligned}
& Y_{\mathrm{F}} Y_{\mathrm{F}}^{\mathrm{T}}=I_{\mathrm{q} \times \mathrm{m}}, \\
& \operatorname{cond}\left(Y_{\mathrm{F}}\right)=1 .
\end{aligned}
$$

where $\operatorname{cond}(\cdot)$ returns the condition number of a given matrix.

To compute the input basis, make $R_{0}=\left(\begin{array}{llll}I_{\mathrm{m}} & I_{\mathrm{m}} & \ldots & I_{\mathrm{m}}\end{array}\right)$ with $I_{\mathrm{m}}$ the identity matrix of order $m$, and the following steps are equivalent. Then, $I_{\mathrm{F}}=\left(\Re\left[I_{\mathrm{F}}^{\mathrm{c}}\right] \Im\left[I_{\mathrm{F}}^{\mathrm{c}}\right]\right)^{1}$.

Step 2 - Orthogonal projection Because we need to extract specific information from the ambient space defined by the response samples, one may be interested in the orthogonal projection.

Now that $Y_{\mathrm{F}}$ and $I_{\mathrm{F}}$ are computed, the orthogonal projection is defined as in (17) because $I_{\mathrm{F}} I_{\mathrm{F}}^{\mathrm{T}}=I_{\mathrm{m} \times \mathrm{q}}$.

$$
H_{\mathrm{F}} / I_{\mathrm{F}}^{\perp}=H_{\mathrm{F}}-H_{\mathrm{F}} I_{\mathrm{F}}^{\mathrm{T}} I_{\mathrm{F}} .
$$

Step 3- The SVD and its partitions

$$
H_{\mathrm{F}} / I_{\mathrm{F}}^{\perp}=U S V^{\mathrm{T}}=\left[\begin{array}{ll}
U_{1} & U_{2}
\end{array}\right]\left[\begin{array}{cc}
S_{1} & 0 \\
0 & 0
\end{array}\right]\left[\begin{array}{c}
V_{1}^{\mathrm{T}} \\
V_{2}^{\mathrm{T}}
\end{array}\right] .
$$

Once the the decomposition is performed, it is possible to determine the order of the system (denoted by $n$ ) from the number of dominant singular values. An interesting decision is to make $\mathrm{n}=\operatorname{rank}(S)$.

Let $\gamma \in \Re^{(\mathrm{p} \times \mathrm{q}) \times \mathrm{n}}$ be:

$$
\gamma=U_{1} S_{1}^{1 / 2}
$$

where $\overline{\bar{\gamma}}$ denotes $\gamma$ without the first 2 p rows; $\bar{\gamma}$ corresponds to $\gamma$ without the first $\mathrm{p}$ rows and without the last $\mathrm{p}$ rows; $\underline{\underline{\gamma}}$ denotes $\gamma$ without the last $2 \mathrm{p}$ rows. Also $\gamma_{\mathrm{p}}$ as the first $\overline{\mathrm{p}}$ rows of $\gamma$.

Step 4- Computing the system matrices $A$ and $C$ In this step, the aforementioned algorithms propose different approaches to compute $A$ and $C$. In Overschee and Moor (1996a) the authors use the information from the SVD as follows

\footnotetext{
1 Note that (15) and (16) holds for the input basis as well.
} 


$$
\begin{gathered}
A=\left[D_{1} \underline{\bar{\gamma}}\right]^{\dagger}\left[\overline{\bar{\gamma}}-D_{2} \underline{\gamma}\right] . \\
C=Z_{0}^{1 / 2} \gamma_{\mathrm{p}} .
\end{gathered}
$$

where $(\cdot)^{\dagger}$ stands for the Moore-Penrose pseudo-inverse, a generalized inverse matrix.

In McKelvey et al. (1996), the authors replace the use of $\gamma$ and its partitions by matrices $J_{1}, J_{2}$ and $J_{3}$, and compute $A$ and $C$ as follows. Differently from (20) and (21), this approach does not use information from matrix $\mathrm{S}$, provided by the SVD.

$$
\begin{array}{r}
A=\left(J_{1} U_{\mathrm{s}}\right)^{\dagger} J_{2} U_{\mathrm{s}} . \\
C=J_{3} U_{\mathrm{s}} .
\end{array}
$$

where

$$
\begin{array}{r}
J_{1}=\left[\begin{array}{ll}
I_{(\mathrm{q}-1) \mathrm{p}} & 0_{(\mathrm{q}-1) \mathrm{p} \times \mathrm{p}}
\end{array}\right], \\
J_{2}=\left[\begin{array}{ll}
0_{(\mathrm{q}-1) \mathrm{p} \times \mathrm{p}} & I_{(\mathrm{q}-1) \mathrm{p}}
\end{array}\right], \\
\\
J_{3}=\left[\begin{array}{ll}
I_{\mathrm{p}} & 0_{\mathrm{p} \times(\mathrm{q}-1) \mathrm{p}}
\end{array}\right] .
\end{array}
$$

where $I_{\mathrm{i}}$ denotes the ith order identity matrix and $0_{\mathrm{i} \times \mathrm{j}}$ denotes the $\mathrm{i} \times \mathrm{j}$ order zeros matrix.

Step 5-Computing the system matrices $B$ and D Lastly, $B$ and $D$ is determined through a least squares solution of (24).

$$
\min _{B, D}\left(L-M\left[\begin{array}{l}
B \\
D
\end{array}\right]\right)
$$

for $L$ and $M$ defined as follows

$$
\begin{gathered}
L=\left(\begin{array}{c}
Y\left(j w_{1}\right) \\
Y\left(j w_{2}\right) \\
\vdots \\
Y\left(j w_{N}\right)
\end{array}\right) \\
M=\left(\begin{array}{cc}
C\left(j w_{1} I_{\mathrm{n}}-A\right)^{-1} & I_{\mathrm{p}} \\
C\left(j w_{2} I_{\mathrm{n}}-A\right)^{-1} & I_{\mathrm{p}} \\
\vdots & \vdots \\
C\left(j w_{\mathrm{N}} I_{\mathrm{n}}-A\right)^{-1} & I_{\mathrm{p}}
\end{array}\right)
\end{gathered}
$$

\section{PRELIMINARY RESULTS}

In this section, numerical examples are presented to corroborate and validate the previously exposed theory. We start this discussion with a comparison on the algorithms in Subsection 4.1, with regard to the fourth step of the algorithm. Then, to illustrate the performance of subspace techniques, we choose two highly resonant system of large order that span some decades of frequency. In both cases, the algorithm described in Subsection 3.1 was applied with number $\mathrm{N}$ of sample frequencies much bigger than $\mathrm{q}$.

The first study case uses simulated data and the model approximation is shown in Subsection 4.2. The second one presents estimation results for real data collected from a resonant beam, in Subsection 4.3. The data set was shared by James S. Welsh from University of Newcastle, Australia.

\subsection{Comparing the algorithms}

At this point the reader may ask what entails the different approaches to compute $A$ and $C$. To answer that, we

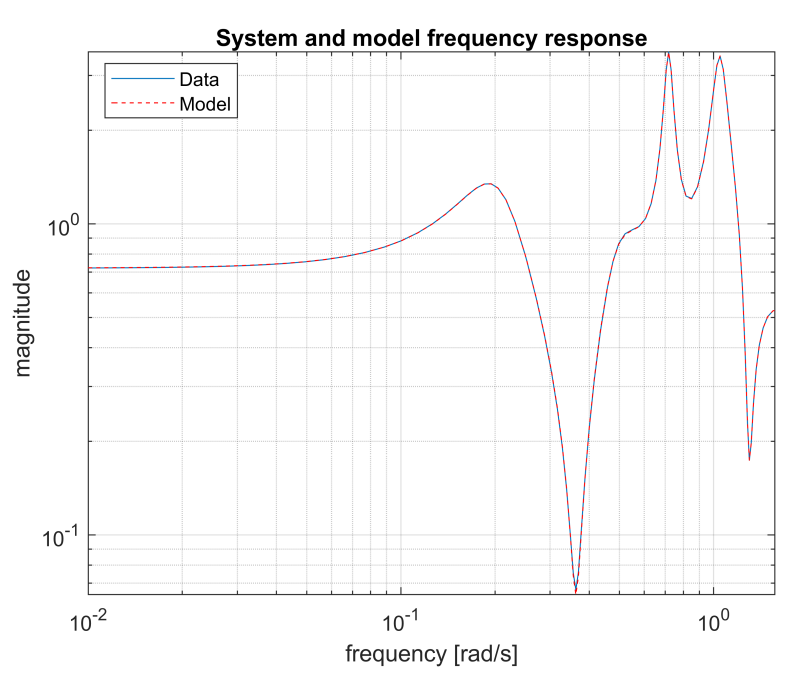

Figure 1. Magnitude of system and model frequency response.

use both methods to estimate a simple system, with the following parameters in regard to the representation in (4), and then check their eigenvalues.

$$
\begin{gathered}
A_{1}=\left[\begin{array}{ccc}
-0.1200 & -9 & 0 \\
1 & 0 & 0 \\
0 & 1 & 0
\end{array}\right], \\
B_{1}=\left[\begin{array}{l}
1 \\
0 \\
0
\end{array}\right], \\
C_{1}=\left[\begin{array}{ccc}
1 & 0.1000 & 7.5000
\end{array}\right], \\
D_{1}=0 .
\end{gathered}
$$

On one hand, the estimation of matrix $C_{1}$ returned exactly the same parameters for the two methods. About matrix $A_{1}$, which eigenvalues are $[0 ; 0.0600+2.9993 i ; 0.0600-$ $2.9993 i]$, until the fourteenth decimal place, there was no difference in the estimated eigenvalues from both methods. Therefore, despite the different approaches, the final estimation may not be affected by choosing one method instead of one another. Taking that in consideration, from this time forth, we prefer to compute $A$ and $C$ via (20) and (21), respectively.

\subsection{Illustrative example}

Consider the noise-free case in which 350 samples were acquired from a synthetic continuous-time single-input single-output system of order 15 , described by a set of state-space equations as in (2) with real $A, B, C$ and $D$ matrices.

The approximation between the model and the system frequency response is illustrated in Fig. 1 and Fig. 2, for magnitude and phase respectively. The Root Mean Square Error (RMSE) computed is approximately $10^{-7}$. Moreover, to this illustrative example whose order was known a priori, the rank of S matrix from SVD provided the expected estimate order.

\subsection{Real data from a resonant beam}

In this topic, in additional to a higher level of resonance, real data from measurement is used to estimate a model. 


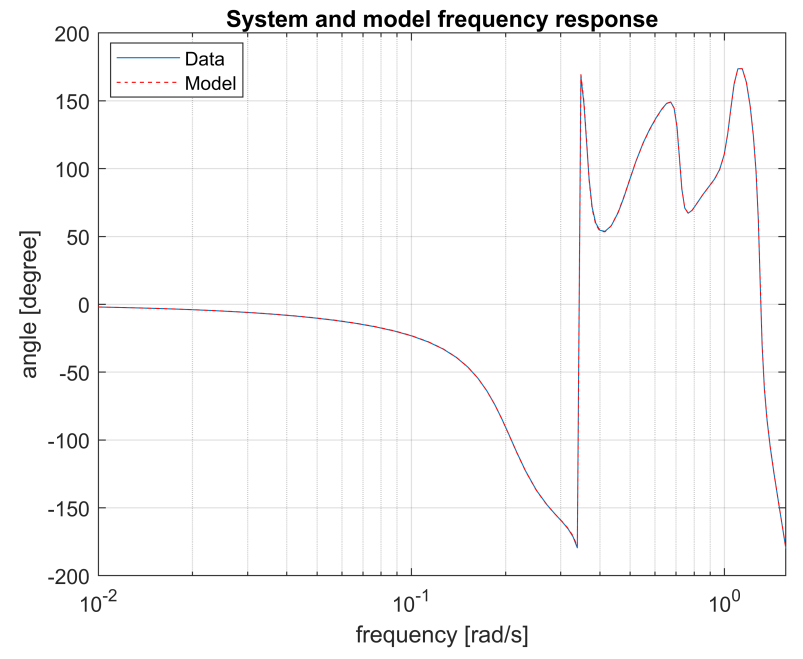

Figure 2. Angle of system and model frequency response.

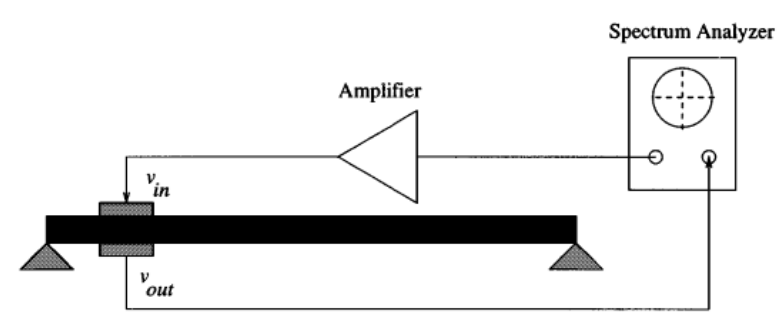

Figure 3. Resonant beam experimental setup.

The experimental setup and measurement samples have been presented in (Welsh and Goodwin, 2003; Gilson et al., 2018), the frequency axis is defined from $10 \mathrm{rad} / \mathrm{s}$ to 600 $\mathrm{rad} / \mathrm{s}$ and 1600 points were used to estimate the model.

Fig. 3 illustrates the experimental setup described in Gilson et al. (2018). The bar consists of a $60 \mathrm{~cm}$ long uniform aluminum beam and a pair of piezoelectric elements are attached symmetrically to either side of the beam. In this experiment, a vector analyzer is used to determine the frequency response of the piezoelectric laminate and the experimental data span approximately two decades.

The data frequency response is illustrated in full line and the approximation is illustrated in in dashed line in Fig. 4 for magnitude, and Fig. 5 for phase.

The estimate model is of $19 t h$ order, and the computed RMSE is approximately 0.4205 , which is considerable. It is possible to note that the algorithm showed more difficulty to approximate a model from real data than simulated data.

Literature shows that in such cases, using real data to estimate parameters, the identification techniques fail in some peaks, specially the low frequency ones. Therefore, as a suggestion for future works, the authors point the necessity to weight the frequency response samples, so that a weighted subspace-based algorithm is developed. This procedure claims to improve efficiency and diminish the RMSE.

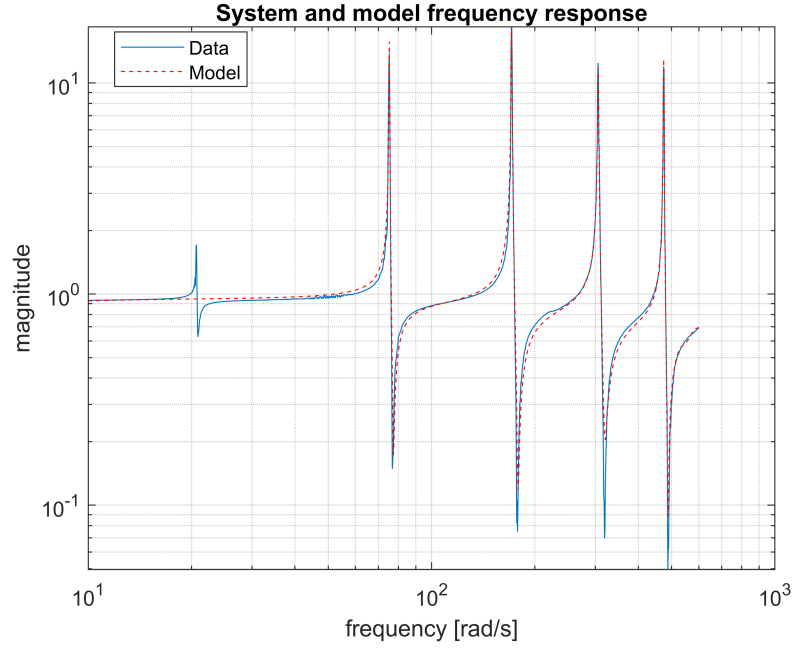

Figure 4. Magnitude of frequency response from resonant beam and model.

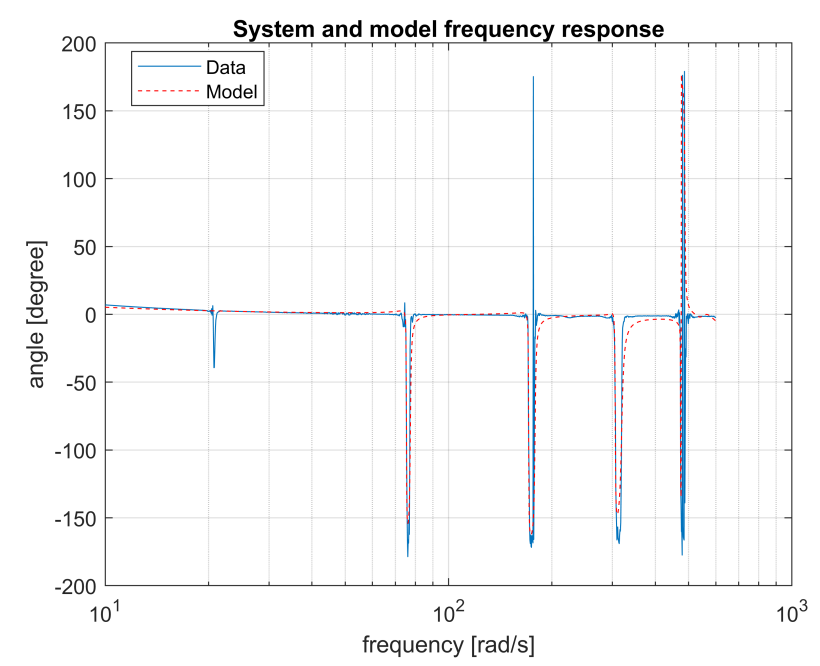

Figure 5. Angle of frequency response from resonant beam and model.

\section{CONCLUSION}

This paper discussed the application of subspace based algorithms to compute a state-space model of highly resonant systems. Two solid algorithms for frequency domain identification were discussed and they showed very similar estimation results. The discussed methodology deals with well conditioned matrices what is an advantage when comparing to other subspace-based procedures. To validate the technique, this paper have examined subspace approach efficiency using a simulated example and real data from a resonant beam. We showed that the research with subspace must forward toward a better approximation of real data, by means of improvements in the algorithm.

\section{ACKNOWLEDGMENT}

The authors are grateful to Prof. James S. Welsh, from Newcastle University, Australia, for having shared the case study measurement data. 


\section{REFERENCES}

Bakir, P.G. (2011). Automation of the stabilization diagrams for subspace based system identification. Expert Systems With Applications, 38, 14390-14397.

Bechara, R. (2010). Análise de falhas de transformadores de potência (in portuguese). Master's thesis, Universidade de São Paulo.

Cigrè-JWG-A2/C4.39 (2014). Electrical transient interaction between transformers and power system. Technical Brochure $577 \mathrm{~A}$ and $577 B$.

Cigrè-JWG-A2/C4.52 (2020). High-frequency transformer and reactor models for network studies-box models. Cigrè technical brochure, (unpublished).

Deschrijver, D., Mrozowski, M., Dhaene, T., and Zutter, D.D. (2008). Macromodeling of multiport systems using a fast implementation of the vector fitting method. IEEE Microwave and Wireless Components Letters, 18, $383-385$.

Dou, S. and Jensen, J.S. (2015). Optimization of nonlinear structural resonance using the incremental harmonic balance method. Journal of Sound and Vibration, 334, $239-254$.

El-Gazzar, D.M. (2017). Finite element analysis for structural modification and control resonance of a vertical pump. Alexandria Engineering Journal, 56(4), 695 707.

Gilson, M., Welsh, J.S., and Garnier, H. (2018). A frequency localizing basis function-based iv method for wideband system identification. IEEE Transactions on Control Systems Technology, 26(1), 329-335.

Gorayan, R. and Chandrakar, K. (2013). Analysis and control of transient enclosure voltages in gis (emtp simulation studies). IEEE India Council International Conference.

Grivet-Talocia, S. and Gustavsen, B. (2016). Passive Macromodeling: Theory and Applications. John Wiley and Sons Inc.

Jeong, Y.J., Park, M.S., You, Y.J., Lee, D.H., and Kim, B.C. (2013). Structural safety and design requirements of cfmp based offshore wind substructure system. In 2013 OCEANS - San Diego, 1-5.

Kumon, T., Iwasaki, M., Suzuki, T., Hashiyama, T., Matsui, N., and Okuma, S. (2000). Nonlinear system identification using genetic algorithm. In 2000 26th Annual Conference of the IEEE Industrial Electronics Society. IECON 2000. 2000 IEEE International Conference on Industrial Electronics, Control and Instrumentation. 21st Century Technologies, volume 4, 2485-2491 vol.4.

Ljung, L. (2007). Frequency domain versus time domain methods in system identification - revisited. Technical report from Automatic Control at Linkopings universitet.

McKelvey, T. (1995). Identification of state-space models from time and frequency data. PhD dissertationLinkoping University.

McKelvey, T., AkCay, H., and Ljung., L. (1996). Subspacebased multivariable system identification from frequency response data. IEEE Transactions on Automatic Control, 41.

Noshadi, A., Shi, J., Lee, W.S., Shi, P., and Kalam, A. (2016). System identification and robust control of multi-input multi-output active magnetic bearing systems. IEEE Transactions on Control Systems Technol- ogy, 24(4), 1227-1239.

Overschee, P. and De Moor, B. (1991). Subspace algorithms for the stochastic identification problem. In [1991] Proceedings of the 30th IEEE Conference on Decision and Control, 1321-1326 vol.2.

Overschee, P.V. and Moor, B.D. (1996a). Continuous-time frequency domain subspace system identification. Signal Processing, 52, 179-194.

Overschee, P.V. and Moor, B.D. (1996b). Subspace Identification for Linear Systems Theory - Implementation Applications. Springer, Boston.

Park, S. and Noh, H.Y. (2011). Updating structural parameters with spatially incomplete measurements using subspace system identification. Expert Systems With Applications, 38 .

Rongshan, Y., Ying, S., and Nambiar, M. (2014). Fast system identification using prominent subspace lms. Digital Signal Processing, 27, 44.

Ruzziconi, L., Younis, M.I., and Lenci, S. (2013). Parameter identification of an electrically actuated imperfect microbeam. International Journal of Non-Linear Mechanics, 57, $208-219$.

Shipp, D., Dionise, T., Lorch, V., and MacFarlane, B. (2011). Transformer failure due to circuit-breakerinduced switching transients. IEEE Transactions on Industry Applications, 47, 707-718.

Shu, Y., Chen, W., Li, Z., Dai, M., Li, C., and v Xianglian Yan, W.L. (2013). Experimental research on very-fast transient overvoltage in $1100-\mathrm{kv}$ gas-insulated switchgear. IEEE Transactions on Power Delivery, 28, 458-466.

Welsh, J.S. and Goodwin, G.C. (2003). Frequency localising basis functions for wide-band identification. In 2003 European Control Conference (ECC), 376-381.

Yao, G. and Li, F. (2015). Stability and nonlinear vibration of an axially moving isotropic beam. In 2015 IEEE International Conference on Cyber Technology in Automation, Control, and Intelligent Systems (CYBER), 1982-1985.

Zhao, Z., Hu, C., and Yin, F. (2018). Failure analysis for vibration stress on ball grid array solder joints. In 2018 19th International Conference on Electronic Packaging Technology (ICEPT), 486-490. 